\title{
Microbial synthesis of hydroxytyrosol and hydroxysalidroside
}

\author{
Hye Jeong $\mathrm{Choo}^{1} \cdot$ Eun Ji Kim ${ }^{1} \cdot$ So Yeon Kim ${ }^{1}$. \\ Youngshim Lee $^{1} \cdot$ Bong-Gyu Kim ${ }^{2} \cdot$ Joong-Hoon Ahn ${ }^{1}$
}

Received: 18 February 2018/Accepted: 6 March 2018/Published online: 15 March 2018

(C) The Korean Society for Applied Biological Chemistry 2018

\begin{abstract}
Plant-derived phenolic compounds, such as hydroxytyrosol and hydroxysalidroside, have a beneficial impact on human health owing to their antioxidant activity. In this study, we used Escherichia coli to synthesize hydroxytyrosol. Tyrosine decarboxylase from Papaver somniferum, tyrosine oxidase from Micrococcus luteus, and 4-hydroxyphenylacetate 3-monooxygenase from E. coli were transformed into the bacterial cell. The resulting transformant successfully synthesized hydroxytyrosol. Furthermore, we used the engineered $E$. coli strains to synthesize $\sim 268.3 \mathrm{mg} / \mathrm{L}$ hydroxytyrosol. Three uridine diphosphate-dependent glycosyltransferases (UGTs), which were previously shown to convert tyrosol into salidroside, were tested to synthesize hydroxysalidroside, and one of UGTs was used to synthesize hydroxysalidroside from hydroxytyrosol. Finally, E. coli harboring this UGT converted approximately $50 \%$ of hydroxytyrosol into hydroxysalidroside.
\end{abstract}

Keywords Hydroxysalidroside - Hydroxytyrosol . Metabolic engineering

Joong-Hoon Ahn

jhahn@konkuk.ac.kr

1 Department of Integrative Bioscience and Biotechnology, Bio/Molecular Informatics Center, Konkuk University, Seoul 05029, Republic of Korea

2 Department of Forest Resources, Gyeongnam National University of Science and Technology, 33 Dongjin-ro, Jinju-si, Gyeongsangnam-do 52725, Republic of Korea

\section{Introduction}

Fruits and vegetables are a rich source of phenolic compounds; for example, coffee provides chlorogenic acid, grapes provide resveratrol, and olives provide tyrosol and hydroxytyrosol. The consumption of these compounds through a daily diet can help prevent several diseases. To this end, plant-derived phenolic compounds were originally considered to possess antioxidant activity. However, recent studies have shown that these natural phenolic compounds have diverse range of biological activities, exhibiting antimicrobial, anticancer, anti-inflammation effects, and aiding in the prevention of cardiovascular diseases [1-3].

Hydroxytyrosol, a major component of olive, is one such phenolic compound that is one of the most powerful natural antioxidants [4]. Hydroxytyrosol has been shown to prevent bone loss and can be used for the treatment of osteoporosis [5]. Moreover, this compound promotes the reduction in low-density lipoprotein $[6,7]$, reduces the agerelated neurodegeneration [8], and has a neuroprotective effect on diabetic neuropathy [9]. Thus, due to its inherent health benefits, hydroxytyrosol is considered a "super" nutrient.

Hydroxysalidroside is the glucoside of hydroxytyrosol, and salidroside is an important compound derived from the Chinese medicinal plant Rhodiola sp [10]. Hydroxysalidroside was first isolated from Picrorhiza scrophulariiflora followed by Lepisorus contortus and is a known antioxidant [11, 12]. Moreover, hydroxysalidroside is reported to exert a neuroprotective effect against $\mathrm{H}_{2} \mathrm{O}_{2}$ and 6-hydroxydopamine [13].

Hydroxytyrosol, classified as a phenylethanoid, has a C6-C2 carbon skeleton and is synthesized from tyrosine. The rational biosynthetic pathway of hydroxytyrosol is as 
follows: decarboxylation and deamination of tyrosine by tyrosine decarboxylase (TDC) and tyramine oxidase (TYO), respectively, leads to the formation of 2-(4-hydroxyphenyl) acetaldehyde. In a microbial system such as Escherichia coli, 2-(4-hydroxyphenyl)acetaldehyde is spontaneously converted into tyrosol. The hydroxylation of tyrosol results in the formation of hydroxytyrosol. Moreover, the conversion of tyrosine into 2,4-dihydroxyphenylalanine (DOPA) by tyrosine hydroxylase (TH) at the initial stage could also lead to the synthesis of hydroxytyrosol [14]. Previously, hydroxytyrosol was synthesized by using $T H$, DOPA decarboxylase, and TYO [15]. To this end, genes required for the synthesis of cofactor tetrahydrobiopterin of $\mathrm{TH}$ have been introduced in $E$. coli to increase the conversion of tyrosine into DOPA. On the other hand, hydroxysalidroside has not been synthesized biologically because no uridine-dependent glycosyltransferase (UGT) has been discovered for the conversion of hydroxytyrosol into hydroxysalidroside.

In this study, we synthesized hydroxytyrosol and hydroxysalidroside in $E$. coli. 4-Hydroxyphenylacetate 3-monooxygenase (HpaBC) from E. coli along with TDC and TYO was employed to synthesize hydroxytyrosol. HpaBC was used to convert tyrosine into L-DOPA [16]. To synthesize hydroxysalidroside, we took the advantage of the structural similarity between tyrosol and hydroxytyrosol to identify a UGT that converts hydroxytyrosol into hydroxysalidroside. Furthermore, by increasing the endogenous tyrosine content in $E$. coli by overexpressing genes involved in tyrosine biosynthesis, we aimed to increase the final yield of hydroxytyrosol and hydroxysalidroside.

\section{Materials and methods}

\section{Constructs and $E$. coli strain}

Tyrosine decarboxylase (TDC from Papaver somniferum; GenBank U08598.1) and tyrosine oxidase (TYO from Micrococcus luteus; GenBank AB010716.1) were synthesized after codon optimization for E. coli (Bioneer, Daejeon, Korea) using the published nucleotide sequences. TDC was subcloned into the BamHI/HindIII site of the pCDF-Duet1 vector (MilliporeSigma, MA, USA), and the resulting construct was named pC-TDC. TYO was introduced into the second cloning site (NdeI/XhoI) of pC-TDC, and the resulting construct was named pC-TDC-TYO.

HpaBC from E. coli (Gene ID: 7156703 and 7155545) was cloned by polymerase chain reaction using $5^{\prime}$ ATGAATTCGATGAAACCAGAAGATTTCCGC-3' (EcoRI restriction site is underlined) and CATGCGGCCGCTTAAATCGCAGCTTCCATT (NotI restriction site is underlined) as primers. The resulting product was subcloned in the EocRI/NotI site of pETDuet1 (MilliporeSigma). pA-aroG-tyrA, pA-aroG ${ }^{\mathrm{fbr}}$ $\operatorname{tyrA}^{\mathrm{fbr}}$, and $\mathrm{pA}$-aroG ${ }^{\mathrm{fbr}}$-ppsA-tktA-tyrA ${ }^{\text {fbr }}$ were cloned previously [17]. The list of constructs is shown in Table 1. The E. coli strain, B-TP, in which both tyrR and pheA were deleted was previously generated [17].

\section{Production and structural determination of reaction products}

Hydroxytyrosol production was carried out according to the protocol described by Kim et al. [17]. Hydroxysalidroside was synthesized using $E$. coli harboring UGT85A1. Hydroxytyrosol was fed into the culture of E. coli harboring UGT85A1. Analysis of reaction products was performed using high performance liquid chromatography (HPLC) [18].

The structure of the reaction product was determined using nuclear magnetic resonance spectroscopy (NMR) [19]. NMR data were as follows; $\delta$ of ${ }^{1} \mathrm{H}$ NMR of hydroxytyrosol in MeOD- $d_{4}(\mathrm{ppm}) ; 6.69(1 \mathrm{H}, \mathrm{d}$, $J=8.0 \mathrm{~Hz}, \mathrm{H}-7), 6.67(1 \mathrm{H}, \mathrm{d}, J=1.7 \mathrm{~Hz}, \mathrm{H}-4), 6.54(1 \mathrm{H}$, $\mathrm{dd}, J=8.0,1.7 \mathrm{~Hz}, \mathrm{H}-8), 3.69(2 \mathrm{H}, \mathrm{t}, J=7.3 \mathrm{~Hz}, \mathrm{H}-1)$, $2.68(2 \mathrm{H}, \mathrm{t}, J=7.3 \mathrm{~Hz}, \mathrm{H}-2)$. $\delta$ of ${ }^{1} \mathrm{H}$ NMR of hydroxysalidroside in DMSO- $d_{6}(\mathrm{ppm}) ; 6.59(1 \mathrm{H}, \mathrm{d}, J=8.0 \mathrm{~Hz}$, H-7), 6.58 (1H, d, $J=2.2 \mathrm{~Hz}, \mathrm{H}-4), 6.44$ (1H, dd, $J=8.0$, $2.2 \mathrm{~Hz}, \mathrm{H}-8), 4.12(1 \mathrm{H}, \mathrm{d}, J=7.8 \mathrm{~Hz}, \mathrm{H}-\mathrm{glc} 1), 3.82(1 \mathrm{H}$, m, H-1a), 3.62 (1H, d, $J=11.5 \mathrm{~Hz}, \mathrm{H}-$ glc6a), $3.51(1 \mathrm{H}, \mathrm{m}$, H-1b), 3.39 (1H, dd, $J=11.7,5.8 \mathrm{~Hz}, \mathrm{H}-$ glc $6 \mathrm{~b}), 3.10(1 \mathrm{H}$, $\mathrm{t}, J=8.8 \mathrm{~Hz}, \mathrm{H}-\mathrm{glc} 2), 3.03-3.06(1 \mathrm{H}, \mathrm{ddd}, J=9.6,5.9$, $2.0 \mathrm{~Hz}, \mathrm{H}$-glc5), $3.00(1 \mathrm{H}, \mathrm{m}, \mathrm{H}$-glc3), $2.92(1 \mathrm{H}, \mathrm{t}$, $J=8.4 \mathrm{~Hz}, \mathrm{H}-\mathrm{glc} 4), 2.63$ (2H, m, H-2).

\section{Results and discussion}

\section{Engineering $E$. coli for the synthesis of hydroxytyrosol}

Hydroxytyrosol is synthesized from tyrosol by a one-step reaction. Conversion of tyrosine into DOPA (3,4-dihydroxyphenylalanine) could lead to the synthesis of hydroxytyrosol. Previous reports have shown that HpaBC from $E$. coli can be used to convert tyrosine into L-DOPA [16]. Thus, we tested whether HpaBC could convert tyrosol into hydroxytyrosol. E. coli expressing HpaBC was used, and it was found that this strain converted tyrosol into hydroxytyrosol (data not shown).

Next, we tested the biosynthesis of tyrosol using TDC and TYO. TDC and TYO were synthesized after codon optimization and subcloned into the pCDF vector. The construct (pC-TDC-TYO) was then transformed into 
Table 1 Plasmids, Escherichia coli strains, and primers used in this study

\begin{tabular}{|c|c|c|}
\hline $\begin{array}{l}\text { Plasmids or } E . \text { coli } \\
\text { strain }\end{array}$ & Relevant properties or genetic marker & $\begin{array}{l}\text { Source or } \\
\text { reference }\end{array}$ \\
\hline \multicolumn{3}{|l|}{ Plasmids } \\
\hline pACYCDuet & $\mathrm{P} 15 \mathrm{~A}$ ori, $\mathrm{Cm}^{\mathrm{r}}$ & Novagen \\
\hline pCDFDuet & CloDE13 ori, Str $^{r}$ & Novagen \\
\hline pA-aroG-tyrA & pACYCDuet carrying aroG and tyrA & {$[17]$} \\
\hline pA-aroG ${ }^{\mathrm{fbr}}-\mathrm{tyr} \mathrm{A}^{\mathrm{fbr}}$ & pACYCDuet carrying feedback inhibition free of $\operatorname{aro} G$ and tyrA & {$[17]$} \\
\hline $\begin{array}{l}\text { pA-aroG }{ }^{\mathrm{fbr}}-\mathrm{pps} \mathrm{A}- \\
\text { tktA-tyrA }^{\mathrm{fbr}}\end{array}$ & pACYCDuet carrying ppsA, $t k t A$, and feedback inhibition free of aroG and tyrA & {$[17]$} \\
\hline pC-TDC-TYO & $\begin{array}{l}\text { pCDFDuet carrying tyrosine decarboxylase (TDC) from Papaver somniferum and tyrosine oxidase } \\
\text { (TYO) from Micrococcus luteus }\end{array}$ & This study \\
\hline pE-HраBC & pET-duet carrying $\mathrm{HpaBC}$ from Escherichia coli & This study \\
\hline \multicolumn{3}{|l|}{ Strains } \\
\hline BL21 (DE3) & $\mathrm{F}^{-}$ompT hsd $S_{B}\left(\mathrm{r}_{\mathrm{B}}^{-} \mathrm{m}_{\mathrm{B}}^{-}\right)$gal dcm lon (DE3) & \\
\hline B-TP & BL21(DE3) $\Delta t y r R:: F R T-\Delta P h e A:: F R T-k^{R}{ }^{R}-F R T[17]$ & {$[17]$} \\
\hline B-TPF & BL21(DE3) $\triangle t y r R:: F R T-\triangle P h e A:: F R T-\triangle f e a B:: F R T-k_{a n}{ }^{R}-F R T$ & {$[21]$} \\
\hline B-TYS1 & BL21 (DE3) harboring pC-TDC-TYO & This study \\
\hline B-TYS2 & BL21 (DE3) harboring pC-TDC-TYO, and pE-HpaBC & This study \\
\hline B-TYS3 & BL21 (DE3) harboring pA-aroG-tyrA, pC-TDC-TYO, and pE-HpaBC & This study \\
\hline B-TYS4 & BL21 (DE3) harboring pA-aroG ${ }^{\mathrm{fbr}}$-tyrA ${ }^{\mathrm{fbr}}$, pC-TDC-TYO, and pE-HpaBC & This study \\
\hline B-TYS5 & B-TP harboring pA-aroG ${ }^{\mathrm{fbr}}$-tyrA ${ }^{\mathrm{fbr}}$, pC-TDC-TYO, and pE-HpaBC & This study \\
\hline B-TYS6 & B-TPF harboring pA-aroG ${ }^{\mathrm{fbr}}-\mathrm{tyrA}^{\mathrm{fbr}}, \mathrm{pC}-\mathrm{TDC}-\mathrm{TYO}$, and pE-HpaBC & This study \\
\hline
\end{tabular}

E. coli (B-TYS1 in Table 1), and the production of tyrosol in B-TYS1 was examined. As shown in Fig. 1A, a new peak which had the retention time similar to a standard tyrosol was observed.

Next, we introduced $H p a B C$ into the B-TYS1 strain and examined the production of hydroxytyrosol using the resulting strain, B-TYS2. The reaction product showed the same HPLC retention time as well as the expected molecular mass of hydroxytyrosol (Fig. 1B). Moreover, the structure of this was determined using proton NMR. It remains unclear as to whether $\mathrm{HpaBC}$ in involved in the conversion of tyrosine into DOPA or the conversion of tyrosol into hydroxytyrosol, or both. However, our goal here was to synthesize hydroxytyrosol.

We attempted to increase the production of hydroxytyrosol in $E$. coli by manipulating the tyrosine biosynthesis pathway in E. coli. Two constructs, both of which contained genes for tyrosine biosynthesis, were co-expressed along with pC-TDC-TYO. The first construct contains 2-dehydro-3-deoxyphosphoheptonate aldolase (aroG) and chorismate mutase/prephenate dehydrogenase (tyrA). AroG is at the entry point of shikimate pathway and catalyzes the formation of 7-phosphate-2-dehydro-3-deoxy-D-arabinoheptulosonate (DAHP) from erythrose-4-phosphate and phosphoenolpyruvate. TyrA catalyzes the conversion of chorismate to prephenate and 4-hydroxyphenylpyruvate, which eventually converts tyrosine by tyrosine aminotransferase (tyrB and aspC). The final product, tyrosine, inhibits aroG and tyrA. Thus, feedback inhibition that was free of aroG and tyrA (pA-aroG ${ }^{\text {fbr }}$-tyrA ${ }^{\text {fbr }}$ ) was used in the second construct [20].

Each construct was transformed into B-TYS2, and the resulting strains $\mathrm{B}-\mathrm{TYS} 3$ and $\mathrm{B}-\mathrm{TYS} 4$ were used for hydroxytyrosol production. B-TYS2-4 produced 22.3, 44.2, and $79.2 \mathrm{mg} / \mathrm{L}$ of hydroxytyrosol, respectively, suggesting that the productivity of hydroxytyrosol was related to the tyrosine content in the cell (Fig. 2A). We also used the E. coli mutant B-TP in which the tyrR and pheA genes were deleted, TyrR represses genes in phenylalanine, tryptophan, and tyrosine biosynthesis, while PheA drives prephenate for phenylalanine synthesis instead of tyrosine. We used another $E$. coli mutant B-TPF, in which feaB was deleted along with tyrR and pheA. FeaB encodes phenylacetaldehyde dehydrogenase which converts 4-hydroxyphenylacetaldehyde into 4-hydroxyphenyllactate instead of tyrosol, thereby lowering the hydroxytyrosol production. $\mathrm{B}-\mathrm{TP}$ and B-TPF mutants were transformed with $\mathrm{pA}$ $\operatorname{aroG}^{\mathrm{fbr}}$-tyrA ${ }^{\mathrm{fbr}}$, pC-TDC-TYO, and pE-HpaBC. The resulting transformants, B-TYS5 and B-TYS6, were tested for hydroxytyrosol production. B-TYS5 produced approximately $239.2 \mathrm{mg} / \mathrm{L}$ and B-TYS6 produced $260.7 \mathrm{mg} / \mathrm{L}$ hydroxytyrosol, both of which were much higher than the 


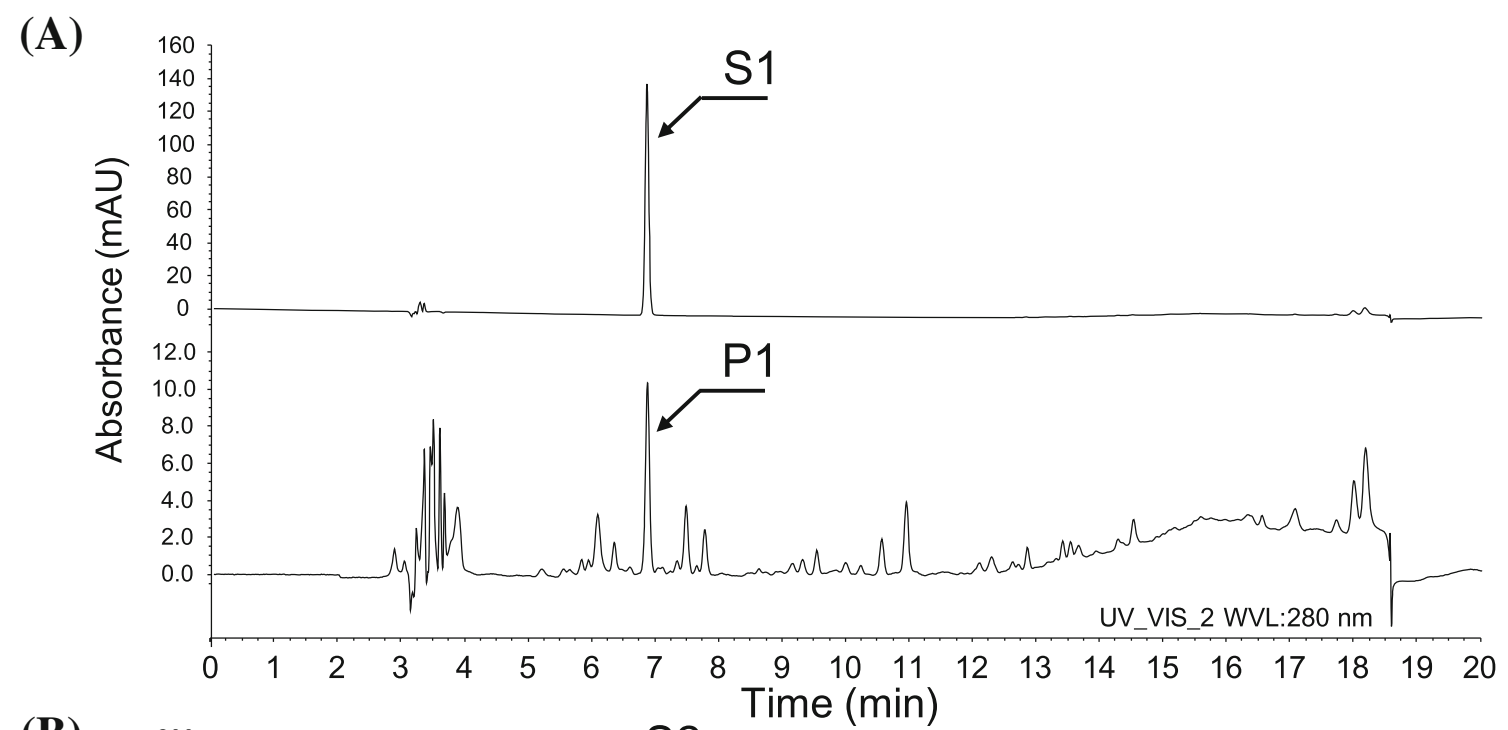

(B)

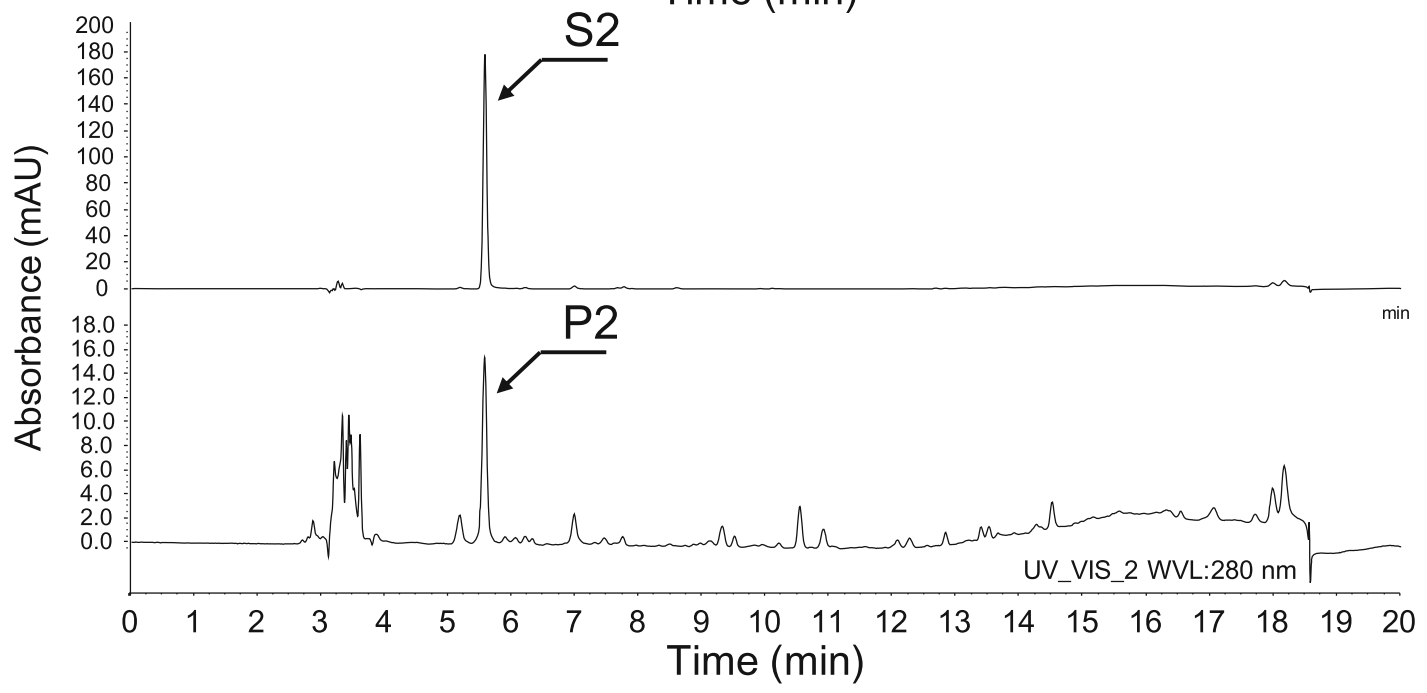

Fig. 1 Synthesis of tyrosol (A) and hydroxytyrosol (B) using B-TYS1 and B-TYS2, respectively. S1, standard tyrosol; P1, reaction product obtained using B-TYS1; S2, standard hydroxytyrosol; P2, reaction product using B-TYS2

Fig. 2 Production of hydroxytyrosol using $E$. coli strains harboring different constructs (A) or E. coli mutants (B)
(A)

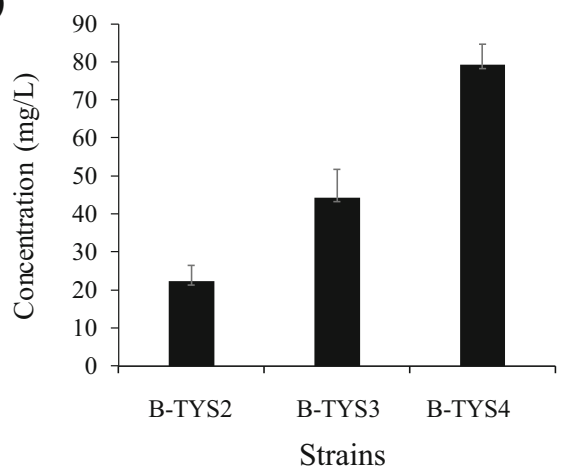

(B)

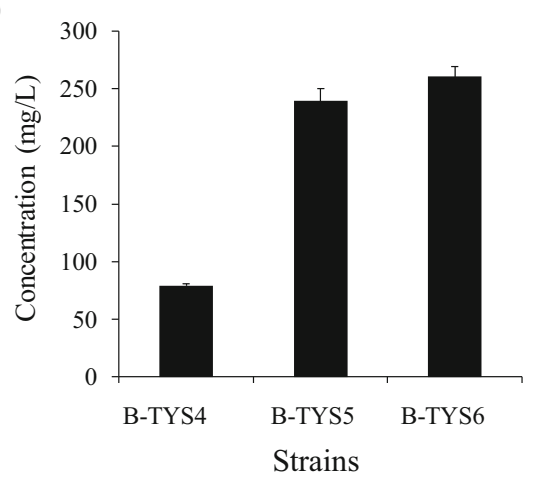

wild type (Fig. 2B). We then monitored the production of hydroxytyrosol using B-TYS6 for $36 \mathrm{~h}$ (Fig. 3). Hydroxytyrosol production increases constantly from 6 to $24 \mathrm{~h}$, while the cells grew from 0 to $12 \mathrm{~h}$. At $30 \mathrm{~h}$, the production of hydroxytyrosol reached $268.3 \mathrm{mg} / \mathrm{L}$. 


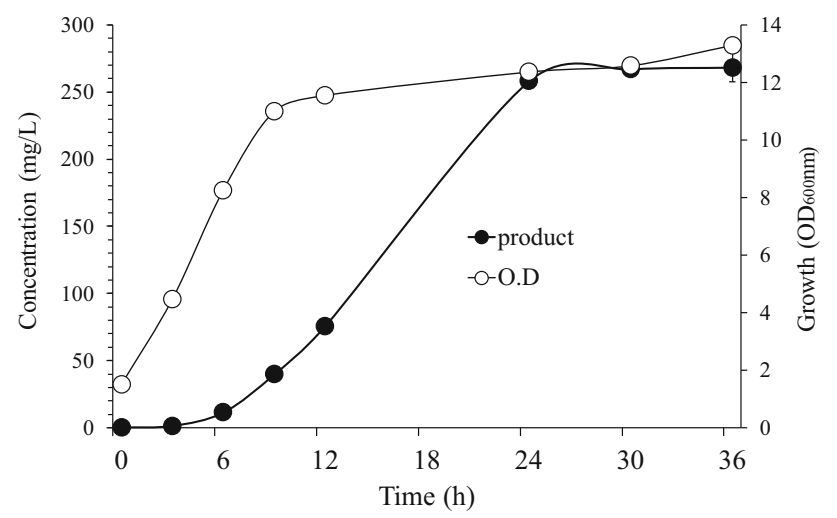

Fig. 3 Monitoring of hydroxytyrosol production using strain B-TYS6

\section{Synthesis of hydroxysalidroside using $E$. coli harboring UGT85A1}

We previously synthesized salidroside from tyrosol using UGT85A1 [21]. Two more UGTs (UGT73C5 and UGT73C6) have also been found to synthesize salidroside from tyrosol. Due to the structural similarity between hydroxytyrosol and tyrosol, these UGTs could transfer glucose from UDP-glucose to hydroxytyrosol to synthesize hydroxysalidroside. Thus, E. coli strains harboring UGT85A1, UGT73C5, or UGT73C6 were used to examine the production of hydroxysalidroside from hydroxytyrosol. After $24 \mathrm{~h}$ of incubation, the culture filtrate from each transformant was analyzed using HPLC. The transformant harboring UGT85A1 showed one new peak, while other two transformants showed two peaks (Fig. 4). However, the mass of both reaction products was $323.809 \mathrm{Da}$, which matched the predicted molecular mass of the compound in which one glucose molecule is attached to hydroxytyrosol. The glucose residue can be attached to either the phenolic hydroxyl group or alcohol group of hydroxytyrosol. Next, we determined the structure of the product obtained from the three E. coli transformants using NMR. NOESY experiments were performed to determine the glycosylation position, and partial NOE correlations are shown in Fig. 5. The glucose moiety was attached at $1-\mathrm{OH}$ as indicated by an NOE cross-peak between H-glc1 and H-1. Therefore, we concluded the structure of the reaction product was that of hydroxysalidroside. E. coli harboring UGT85A1 converted approximately $45 \%$ of hydroxytyrosol into hydroxysalidroside. E. coli harboring UGT73C6 produced more by-product (2-glucosyloxy-4-(2-hydroxylethyl) phenol or 2-glucosyloxy-5-(2-hydroxylethyl)
Fig. 4 Biological synthesis of hydroxysalidroside using $E$. coli harboring UGT85A1 (A), UGT73C5 (B), or UGT73C6 (C). P1 was determined to be hydroxysalidroside. P2 is likely to be (2-hydroxy-5hydroxyethyl)phenyl- $O-\beta$-Dglucopyranoside or (2-hydroxy4-hydroxyethyl)phenyl- $O$ - $\beta$-Dglucopyranoside

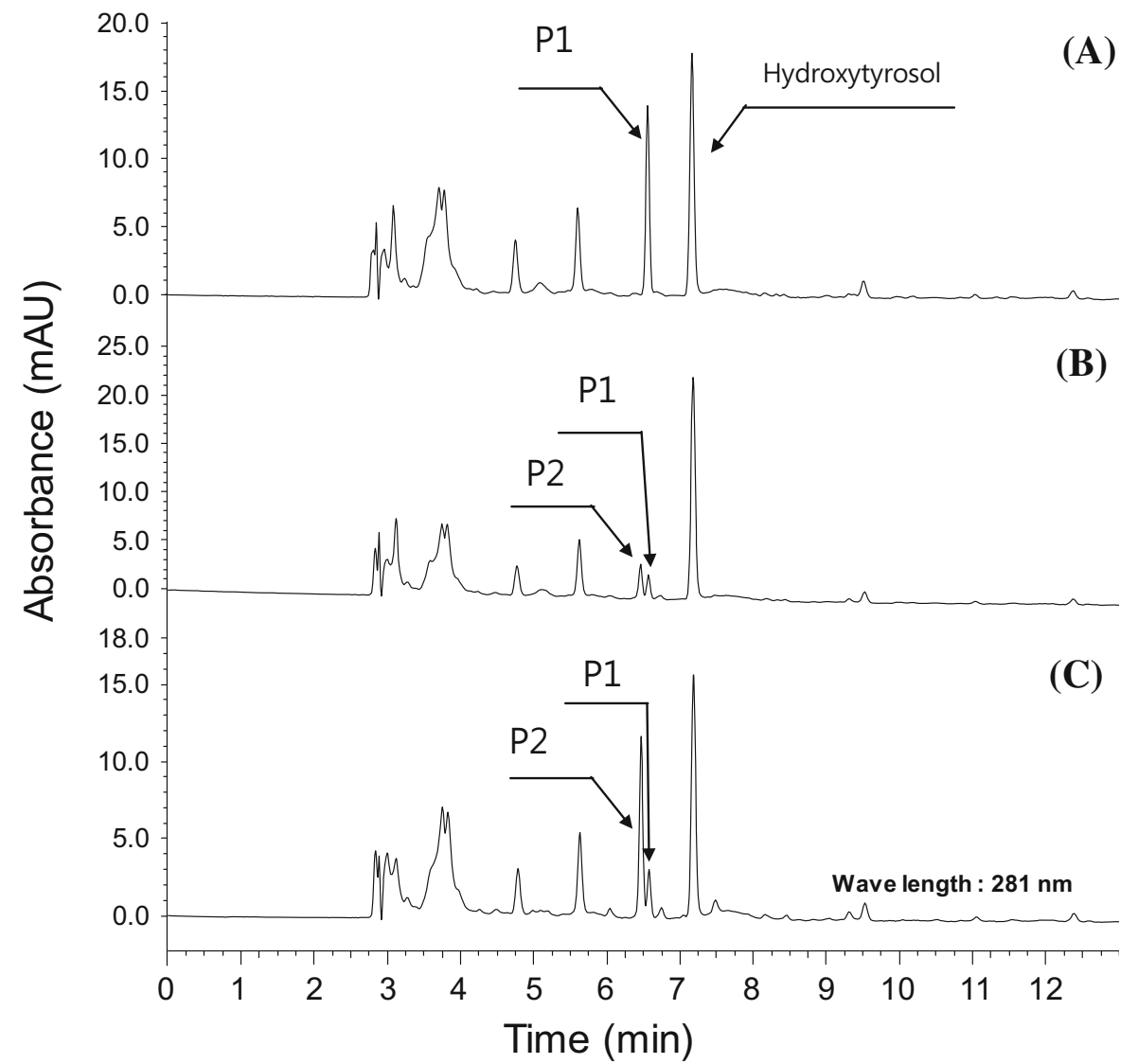


Fig. 5 (A) Partial NOE correlations marked with arrows. The cross-peak between $\mathrm{H}-1$ and $\mathrm{H}-\mathrm{glc} 1$ in the NOESY spectrum clarified the hydroxysalidroside structure. (B) Partial NOESY spectrum showing important cross-peaks to determine the structure of the reaction product

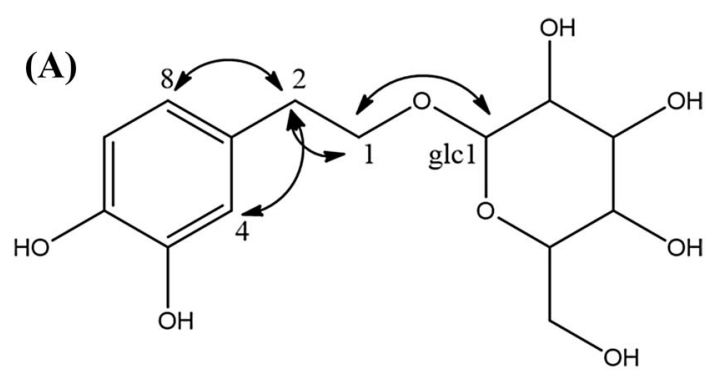

(B)

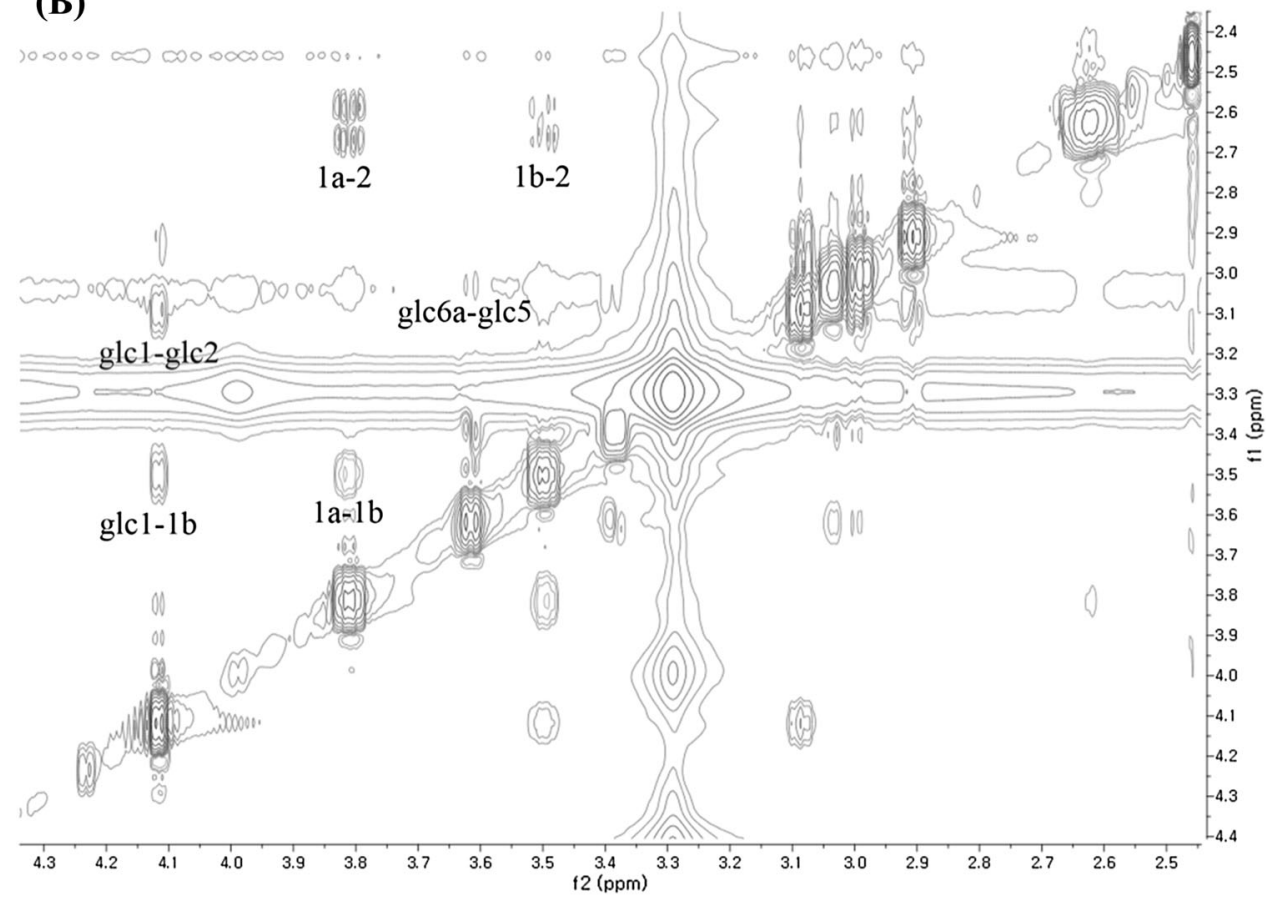

phenol than hydroxysalidroside. The ratio of by-product to hydroxysalidroside was 2:8 with an overall conversion rate of $45 \%$. E. coli harboring UGT73C5 converted only $15 \%$ of hydroxytyrosol into two products, while the by-product was produced to greater extent.

Next, we transformed UGT85A1 into E. coli B-TYS6 and the resulting transformant was used to examine hydroxysalidroside production, but observed no production of hydroxysalidroside. However, because salidroside was synthesized, we speculated that the glycosylation step was inhibited by hydroxylation and examined whether hydroxysalidroside could be synthesized from tyrosol. E. coli harboring HpaBC and UGT85A1 was used to synthesize hydroxysalidroside from tyrosol. This $E$. coli did not convert tyrosol into hydroxysalidroside, producing only hydroxytyrosol. It seemed that metabolite(s) inhibits UGT85A1 during this reaction. Therefore, two-step reactions were used for the synthesis of hydroxysalidroside. We synthesized hydroxytyrosol using B-TYS6, and the filtrate was then extracted and mixed with E. coli harboring UGT85A1. Using this approach, $\sim 45 \%$ of hydroxytyrosol was converted into hydroxysalidroside.
Previous reports have shown that tyrosine hydroxylase instead of HpaBC along with TDC and TYO was used to produce hydroxytyrosol [15]. TDC and TYO recycle cofactor for tyrosine hydroxylase and synthesized $\sim 29.3 \mathrm{mg} / \mathrm{L}$ hydroxytyrosol (when $1 \mathrm{mM}$ tyrosine was fed) or $12.3 \mathrm{mg}$ without tyrosine. We used the HpaBC and tried to supply more tyrosine in $E$. coli by engineering the tyrosine biosynthetic pathway. Through these strategies, we could synthesize $\sim 268.3 \mathrm{mg} / \mathrm{L}$ hydroxytyrosol. Jung et al. [21] also synthesized hydroxytyrosol using $A A S$ (aromatic aldehyde synthase), which is bifunctional, possessing both TDC and TYO activities [22]. Using AAS and HpaBC, we managed to synthesize $208 \mathrm{mg} / \mathrm{L}$ hydroxytyrosol. Thus, for the synthesis of hydroxytyrosol, the current system was better than the previously system that used $A A S$ and $H p a B C$.

Due to the structural similarity between tyrosol and hydroxytyrosol, we used three UGTs (UGT73C5, UGT73C6, and UGT85A1) that converted tyrosol to salidroside. These three UGTs regioselectively synthesized salidrosides from tyrosol [22]. UGT85A1 regioselectively synthesized hydroxysalidroside, while UGT73C5 and 
UGT73C6 produced not only hydroxysalidroside but also other by-product (probably (2-hydroxy-5-hydroxyethyl)phenyl- $O$ - $\beta$-D-glucopyranoside or (2-hydroxy-4-hydroxyethyl)phenyl- $O-\beta$-D-glucopyranoside). The natural substrate of these three UGTs differed significantly. UGT73C6 is known to transfer a glucose residue to 7-OH group of kaempferol 3-O-rhamnoside and quercetin 3-Orhamnoside [23]. UGT73C5 used brassinosteroid as a substrate [24], while UGT85A1 is involved in cytokinin metabolism in Arabidopsis thaliana [25]. These three UGTs have a broad substrate range and differing regioselectivity, which made it possible to synthesize hydroxysalidroside or other glucosylate compounds.

Acknowledgments This work was supported by a grant from the Next-Generation BioGreen 21 Program (PJ01326001), Rural Development Administration, and Priority Research Centers Program through the National Research Foundation of Korea funded by the Ministry of Education, Science, and Technology (2009-0093824).

\section{References}

1. Delmas D, Aires V, Limagne E, Dutartre P, Mazué F, Ghiringhelli F, Latruffe N (2011) Transport, stability, and biological activity of resveratrol. Ann N Y Acad Sci 1215:48-59

2. Upadhyay R, Mohan Rao LJ (2013) An outlook on chlorogenic acids-occurrence, chemistry, technology, and biological activities. Crit Rev Food Sci Nutr 53:968-984

3. Vilaplana-Pérez C, Auñón D, García-Flores LA, Gil-Izquierdo A (2014) Hydroxytyrosol and potential uses in cardiovascular diseases, cancer, and AIDS. Front Nutr 1:18

4. Richards KH (2014) The most powerful natural antioxidant discovered to date-hydroxytyrosol. https://www.prohealth.com/ library/the-most-powerful-natural-antioxidant-discovered-to-date hydroxytyrosol-29641

5. Hagiwara K, Goto T, Araki M, Miyazaki H, Hagiwara H (2011) Olive polyphenol hydroxytyrosol prevents bone loss. Eur J Pharmacol 662:78-84

6. González-Santiago M, Martín-Bautista E, Carrero JJ, Fonollá J, Baró L, Bartolomé MV, Gil-Loyzaga P, López-Huertas E (2006) One-month administration of hydroxytyrosol, a phenolic antioxidant present in olive oil, to hyperlipemic rabbits improves blood lipid profile, antioxidant status, and reduces atherosclerosis development. Atherosclerosis 188:35-42

7. Vázquez-Velasco M, Esperanza Díaz L, Lucas R, Gómez-Martínez S, Bastida S, Marcos A, Sánchez-Muniz FJ (2011) Effects of hydroxytyrosol-enriched sunflower oil consumption on CVD risk factors. Br J Nutr 105:1448-1552

8. Schaffer S, Müller WE, Eckert GP (2010) Cytoprotective effects of olive mill wastewater extract and its main constituent hydroxytyrosol in PC12 cells. Pharmacol Res 62:322-327

9. Ristagno G, Fumagalli F, Porretta-Serapiglia C, Orrù A, Cassina C, Pesaresi M, Masson S, Villanova L, Merendino A, Villanova A, Cervo L, Lauria G, Latini R, Bianchi R (2012) Hydroxytyrosol attenuates peripheral neuropathy in streptozotocin-induced diabetes in rats. J Agric Food Chem 60:5859-5865

10. Yousef GG, Grace MH, Cheng DM, Belolipov IV, Raskin I, Lila MA (2006) Comparative phytochemical characterization of three Rhodiola species. Phytochemistry 67:2380-2391

11. Greca MD, Ferrara Maria, Fiorentino MFA, Monaco P, Previtera L (1998) Antialgal compounds from Zantedeschia aethiopica. Phytochemistry 49:1299-1304

12. Yang JH, Kondratyuk TP, Jermihov KC, Marler LE, Qiu X, Choi Y, Cao H, Yu R, Sturdy M, Huang R, Liu Y, Wang LQ, Mesecar $\mathrm{AD}$, van Breemen RB, Pezzuto JM, Fong HH, Chen YG, Zhang HJ (2011) Bioactive compounds from the fern Lepisorus contortus. J Nat Prod 74:129-136

13. Liu YG, Li X, Xiong DC, Yu B, Pu X, Ye XS (2015) Synthetic phenylethanoid glycoside derivatives as potent neuroprotective agents. Eur J Med Chem 5:313-323

14. Daubner SC, Le T, Wang S (2011) Tyrosine hydroxylase and regulation of dopamine synthesis. Arch Biochem Biophys 508:1-12

15. Satoh Y, Tajima K, Munekata M, Keasling JD, Lee TS (2012) Engineering of L-tyrosine oxidation in Escherichia coli and microbial production of hydroxytyrosol. Met Eng 14:603-610

16. Wei T, Cheng B-Y, Liu J-Z (2016) Genome engineering Escherichia coli for L-DOPA overproduction from glucose. Sci Rep 6:30080

17. Kim MJ, Kim B-G, Ahn J-H (2013) Biosynthesis of bioactive $O$ methylated flavonoids in Escherichia coli. Appl Microbiol Biot 97:7195-7204

18. An DG, Yang SM, Kim BG, Ahn J-H (2016) Biosynthesis of two quercetin $O$-diglycosides in Escherichia coli. J Ind Microbioi Biotechnol 43:841-849

19. Yoon J-A, Kim B-G, Lee WJ, Lim Y, Chong Y, Ahn J-H (2012) Production of a novel quercetin glycoside through metabolic engineering of Escherichia coli. Appl Environ Microbiol $78: 4256-4262$

20. Lütke-Eversloh T, Stephanopoulos G (2007) L-Tyrosine production by deregulated strains of Escherichia coli. Appl Microbiol Biotechnol 75:103-110

21. Jung DE, Kim SY, Ahn J-H (2017) Production of three phenylethanoids, tyrosol, hydroxytyrosol, and salidroside, using plant genes expressing in Escherichia coli. Sci Rep 7:2578

22. Kaminaga Y, Schnepp J, Peel G, Kish CM, Ben-Nissan G, Weiss D, Orlova I, Lavie O, Rhodes D, Wood K, Porterfield DM, Cooper AJ, Schloss JV, Pichersky E, Vainstein A, Dudareva N (2006) Plant phenylacetaldehyde synthase is a bifunctional homotetrameric enzyme that catalyzes phenylalanine decarboxylation and oxidation. J Biol Chem 281:23357-23366

23. Jones P, Messner B, Nakajima J, Schäffner AR, Saito K (2003) UGT73C6 and UGT78D1, glycosyltransferases involved in flavonol glycoside biosynthesis in Arabidopsis thaliana. J Biol Chem 278:43910-43918

24. Poppenberger B, Fujioka S, Soeno K, George GL, Vaistij FE, Hiranuma S, Seto H, Takatsuto S, Adam G, Yoshida S, Bowles D (2005) The UGT73C5 of Arabidopsis thaliana glucosylates brassinosteroids. Proc Natl Acad Sci 102:15253-15258

25. Jin SH, Ma XM, Kojima M, Sakakibara H, Wang YW, Hou BK (2013) Overexpression of glucosyltransferase UGT85A1 influences trans-zeatin homeostasis and trans-zeatin responses likely through $O$-glucosylation. Planta 237:991-999 